\title{
Are inclined air showers from cosmic rays the most suitable to radio detection?
}

\author{
Mohammad Sabouhi ${ }^{1}$ \\ Department of Physics, Semnan University \\ Semnan, Iran \\ E-mail: m.sabouhi@semnan.ac.ir
}

\section{Gohar Rastegarzadeh}

Department of Physics, Semnan University

Semnan, Iran

E-mail: grastegardsemnan.ac.ir

There are many discussions regarding the best possible type of air showers to radio detection. In this study and based on CORSIKA and CoREAS simulations, we investigate the best type of cosmic ray air showers especially with regard to experimental purposes. Two types of air showers are under considerations, Vertical and Inclined. We compare raw radio pulses and filtered peak radio amplitude patterns for different electric field components in the 32 to $64 \mathrm{MHz}$ frequency band using a specially designed computer code to investigate a series of vertical and inclined air showers initiating from proton and Iron primary particles with $10^{17}$ to $10^{18} \mathrm{eV}$ energy ranges. It is found that despite having smaller values in specific distances from shower core, inclined air showers provide more expanded and consistent radio signals and peak radio amplitude values throughout an entire radio detection area compared to vertical air showers. Moreover inclined air showers provide more time window for radio detection which is very important in real experiments.

The 34th International Cosmic Ray Conference

30 July- 6 August, 2015

The Hague, The Netherlands

\section{${ }^{1}$ Speaker}




\section{Introduction}

There is a long history in discovery and investigation of cosmic ray air showers [1]. From real experiments to computer simulations, there has been always a scientific prowling in this field of science. Although there are many available methods to detect cosmic ray air showers, there has always been a search for a more complete and consistent approach in order to investigate cosmic ray air showers. This new technique should have been compatible to be used for studying ultra-high energy cosmic rays, be more independent from environmental changes and complement the existing particle detectors and air fluorescence telescopes all with nearly $100 \%$ duty cycle.

In recent years there has been a significant progress in radio detection of cosmic ray air showers [2],[3],[4],[5]. New experiments [6],[7],[8] with more advanced computer simulation software [9],[10] and the agreements between the two [11] has created a hope to establish a new approach with mentioned features. Earlier analyses have shown that it is possible to drive some of the key parameters of an air shower from radio measurement only [12[]. It is possible to even determine an air shower propagation direction based on radio signal patterns [13]. However there is always a question which needs to be addressed. What type of cosmic ray air showers are the most suitable to radio detection? In this study we analyze two type of air showers by using radio detection technique and Monte Carlo simulations of geosynchrotron radio emission [14]. Air showers can be classified into two main groups, vertical and inclined. In the following we first describe the methodology throughout the study and then start our investigation on vertical followed by inclined air showers.

\section{Methodology}

We use CORSIKA 7.4 [15] and CoREAS 1.0 [16] software to simulate cosmic ray air showers in this study. In order to do a fine investigation on cosmic ray air showers a variety of air showers with $10^{17} \mathrm{eV}$ and $10^{18} \mathrm{eV}$ energy initiating from proton and Iron primary particles were simulated. We use QGSJETII-03 [17],[18] as the hadronic interaction model and thinning set to $10^{-6}$ as recommend in COREAS to generate reasonable data. The earth magnetic field parameter which is very important in radio analyses and can even change radio signal patterns dramatically as we will discuss in an upcoming study is set to correspond with the Pierre Auger Observatory in Argentina [19]. Two sets of radio arrays have been used in this study. First and in order to investigate filed strength values as we move away from shower core, we use a radio array consist of 13 radio antennas located from the shower core to $600 \mathrm{~m}$ in the north direction at the height of $1400 \mathrm{~m}$ above sea level. For the second step and in order to analyze peak radio amplitude values in an entire radio detection area, we use a radio array consist of 73 radio antennas expanded from shower core to $100 \mathrm{~m}$ with 15 degree intervals to cover the entire area. The raw radio data from simulated air showers have been converted and analyzed with a specially designed computer code to obtain the peak radio amplitude values in the $32-64 \mathrm{MHz}$ frequency band for all electric filed components. In the following we first investigate time-dependent raw radio pulses at the center and $100 \mathrm{~m}$ away from shower core which belongs to vertical and inclined air showers with $10^{17} \mathrm{eV}$ and $10^{18} \mathrm{eV}$ initial energy followed by an investigation on radio signal patterns in an entire radio detection area. 


\section{Time-dependent results}

The output data from CoREAS even in its simplest form can reveal some great information about an air shower. In this section we start our analyze by looking at the raw radio pulses obtained from CoREAS and CORSIKA simulations for vertical air showers initiating from proton and Iron primary particles with $10^{17} \mathrm{eV}$ initial energy at the center and $100 \mathrm{~m}$ from shower core in the North direction.
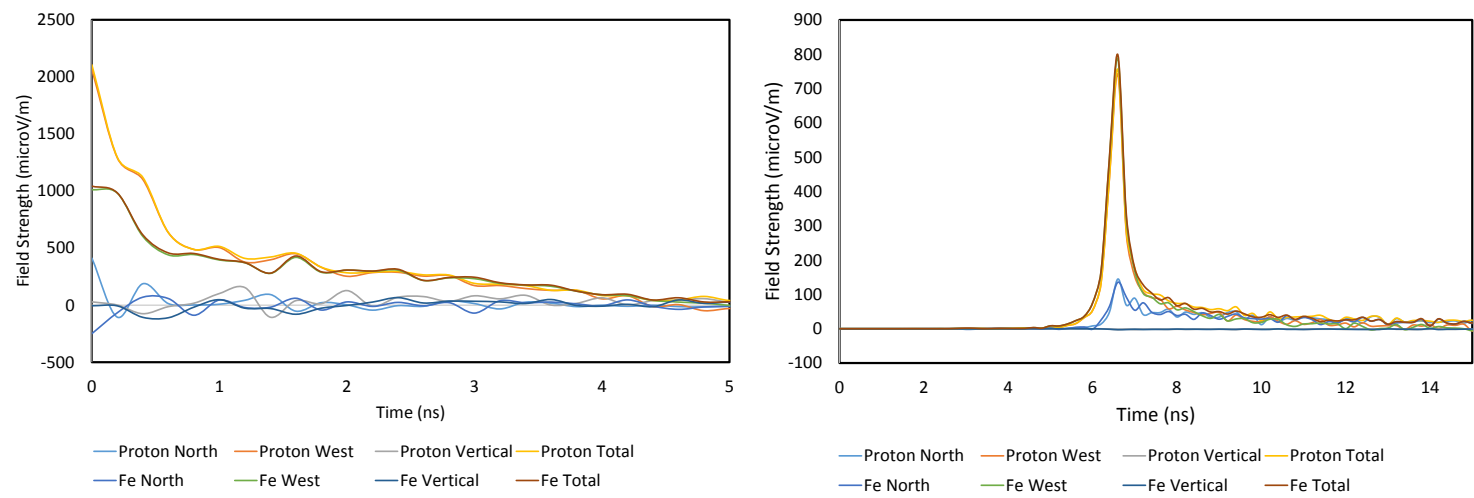

Figure1: From left to right : Raw radio pulses for vertical air showers with $10^{17} \mathrm{eV}$ energy from proton and Iron primary particles as seen at the center and $100 \mathrm{~m}$ from shower core.

As it can be seen in Fig. 1 beside the fact that we always get the strongest radio pulses in the East-West direction due to the East-West effect from earth magnetic field [20] for a shower with this specific property, the decline in the values is very sensible. As we move away from shower cores the maximum measured value drops from 2000 to $800 \mathrm{microV} / \mathrm{m}$ which shows a significant $60 \%$ decrease for the total field strength. It should be noted that due to natural interferences, weak radio pulses may not be very useful since they will be infected by environmental noises [21].
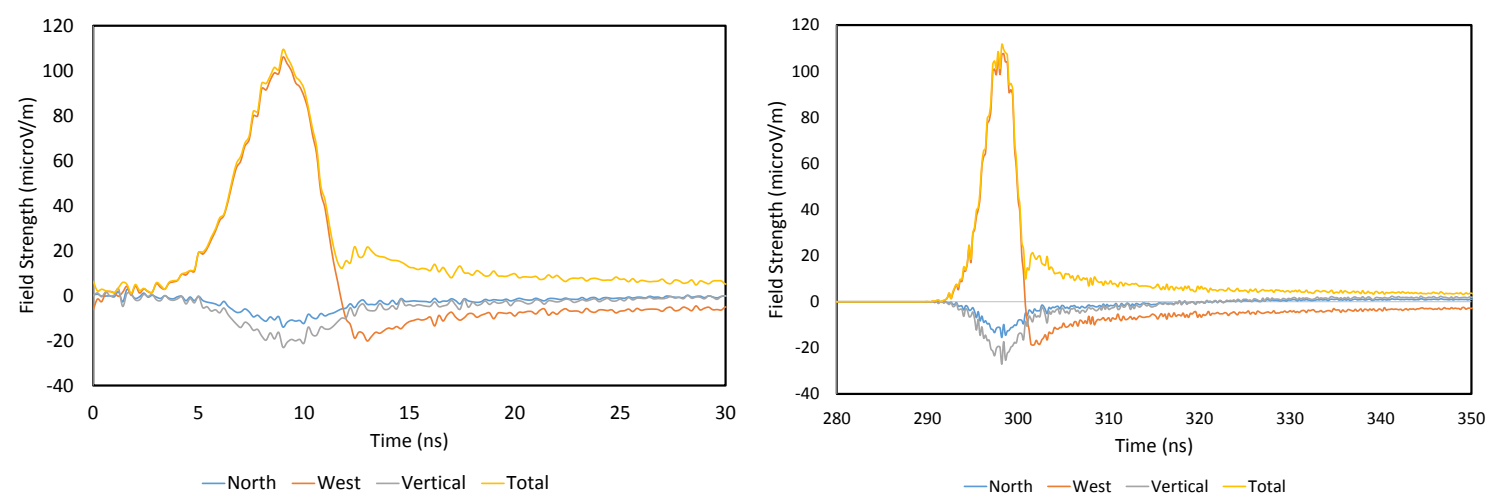

Figure2: From left to right: Raw radio pulses for an inclined proton air shower with $\theta=60^{\circ}$ and $10^{17} \mathrm{eV}$ energy propagating to the North direction as seen at the center and $100 \mathrm{~m}$ from shower core. 
In the other hand and as the Fig. 2 shows, inclined air showers behave in a completely different way. For inclined showers with $10^{17} \mathrm{eV}$ energy initiating from proton primary particle, radio antennas have measured almost the same values for all components especially the West and total field strength at the center and $100 \mathrm{~m}$ from shower core. This is extremely important in real experiments since we may not be able to establish radio arrays right next to the center of an air shower.

Moreover the inclined air showers are more favorable from time perspective too. Comparing Fig. 1 and Fig. 2 shows over $250 \%$ increase in available time window to detect reasonable radio pulses from an inclined compared to a vertical air shower. Although it's clear that we may get weaker signals from inclined air showers but this can be less of a problem if we are looking for ultra-high energy cosmic rays.
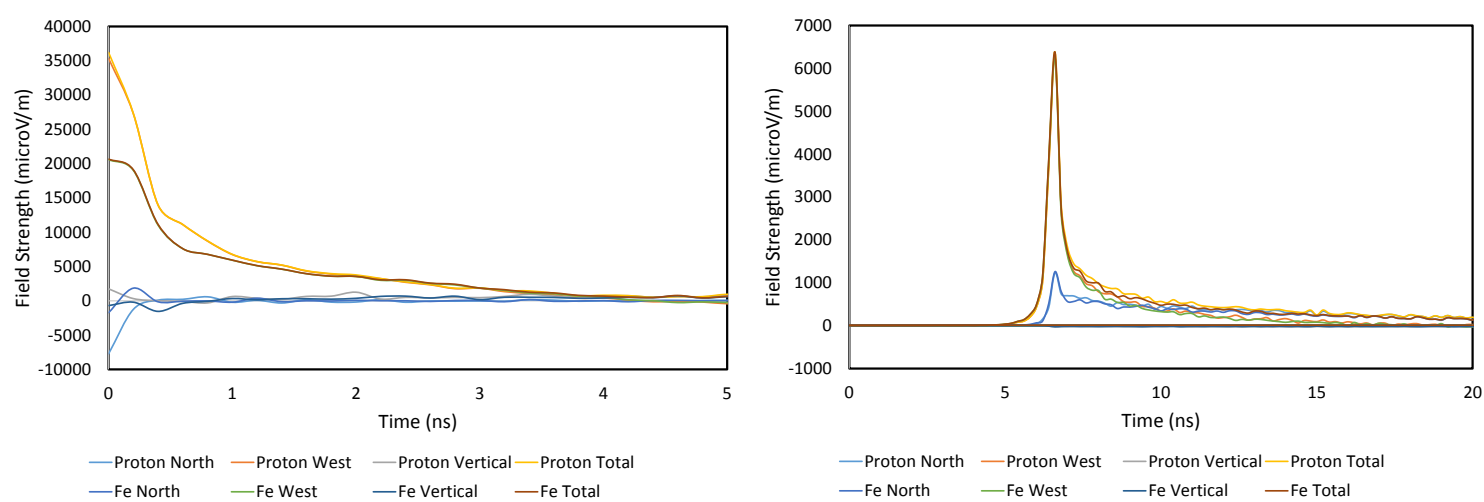

Figure3: From left to right : Raw radio pulses for vertical air showers with $10^{18} \mathrm{eV}$ energy from proton and Iron primary particles as seen at the center and $100 \mathrm{~m}$ from shower core.

Investigating ultra-high energy cosmic ray air showers makes radio detection technique even more applicable. Fig. 3 shows that for vertical showers with $10^{18} \mathrm{eV}$ energy we can expect to have over an order of magnitude increase in radio signal pulses. As we expect, by moving away from shower core to $100 \mathrm{~m}$, the maximum radio signal drops nearly $80 \%$ which shows a significant decrease from 35000 to $6000 \mathrm{microV} / \mathrm{m}$ but it is still much stronger than what radio antennas have detected at the center of a vertical air shower with $10^{17} \mathrm{eV}$ energy for total field component.

Although a sharp decline in the radio amplitude values happens very fast regardless of the initial energy of a vertical shower, there is a good change to measure useful radio signals in a much wider time window when we are dealing with an inclined air shower.

\section{Filtered Peak Radio Amplitude Patterns}

Same scenario happens if we investigate radio signals from vertical and inclined air showers in an entire radio detection area. For this purpose we measure peak radio amplitudes from all electric filed components in the North, West and Vertical directions in order to calculate total field component values for a specific air shower. This is done by using a specially designed computer code by which we'll be able to determine the peak radio amplitude values in the desired frequency band which in this case is $32-64 \mathrm{MHz}$. 
The obtained peak radio amplitude values from different electric fields components can be used to produce $2 \mathrm{D}$ type radio signal patterns. In the following we investigate these patterns for vertical and inclined air showers with $10^{17} \mathrm{eV}$ energy initiating from proton primary particle.
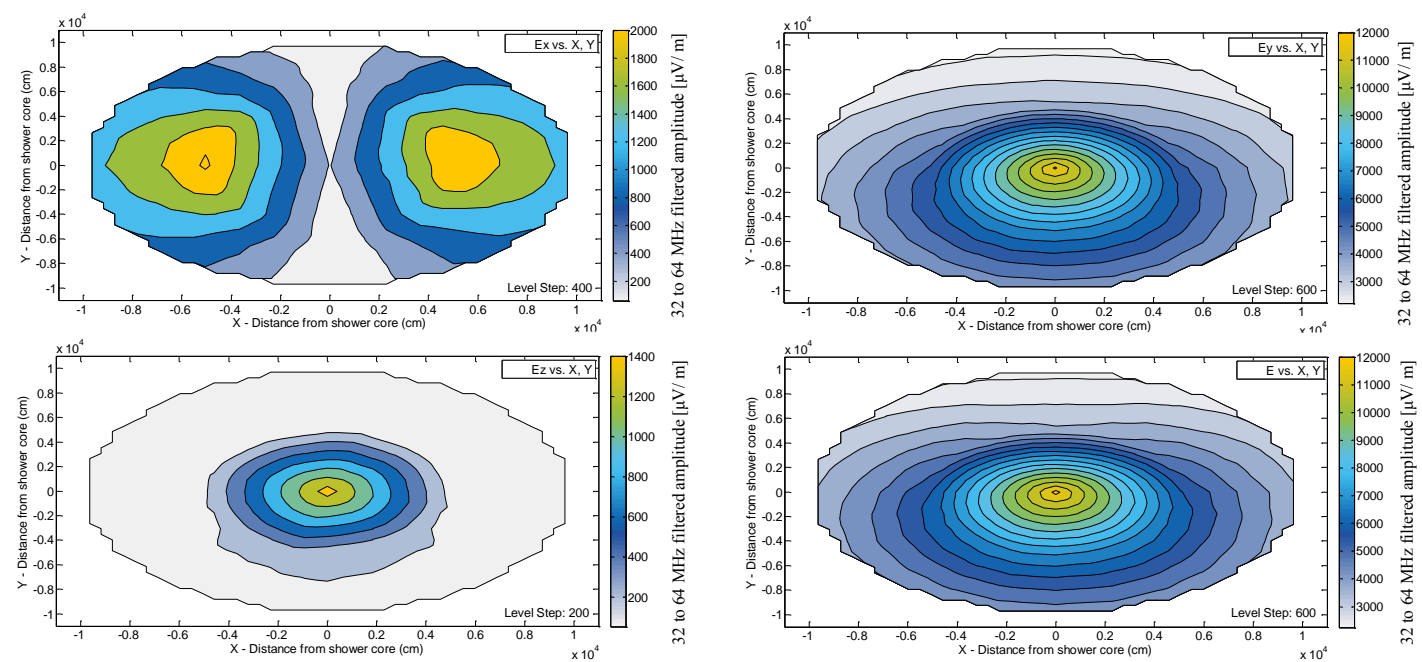

Figure4: From top left to bottom right: Peak radio amplitude patterns of the North, West, Vertical and Total electric filed components for a vertical proton air shower with $10^{17} \mathrm{eV}$ energy.

It is important to investigate the behavior of peak radio amplitude values not only in one direction and in all frequencies as we did in the previous section but also in a specific frequency band throughout an entire radio detection area. Fig.4 shows the radio signal patterns for a vertical shower with $10^{17} \mathrm{eV}$ energy initiating from proton primary particle. Since the West part is still the strongest component, the total field pattern is heavily affected by that shape so these two patterns are almost identical for a shower with this property. This changes when we investigate inclined air showers as other components do have an impact in total field pattern too.
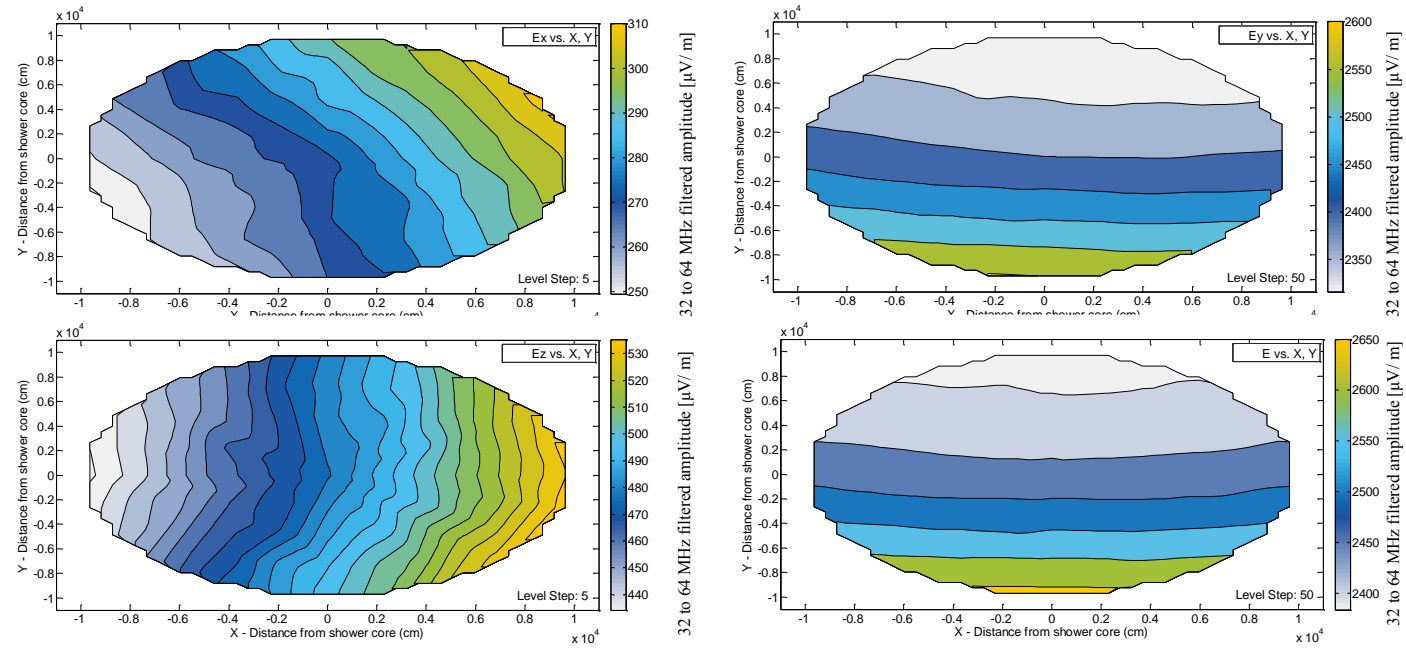

Figure5: From top left to bottom right: Peak radio amplitude patterns of the North, West, Vertical and Total electric filed components for a proton inclined air shower with $\theta=60^{\circ}, 10^{17} \mathrm{eV}$ energy propagating to the North. 
The important point though is the decrease of radio signals as we move away from shower core. Except the radio signal pattern of the electric filed component in the North direction $\left(E_{x}\right)$, by moving $100 \mathrm{~m}$ from shower core we observe a sharp decline in the measured peak radio amplitude values. The total filed component values decreases from the maximum 12000 to $4000 \mathrm{microV} / \mathrm{m}$ which shows nearly $60 \%$ decrease just by moving 100 meter from shower core. It's even more for the vertical electric filed component $\left(E_{z}\right)$ pattern as it drops nearly $90 \%$ just by moving $50 \mathrm{~m}$ from shower core.

It all changes as we investigate inclined air showers. Fig. 5 shows radio signal patterns for an inclined proton air shower propagating to the north with $10^{17} \mathrm{eV}$ initial energy. It is obvious from these patterns that we can expect to measure even stronger values for $E_{x}$ and $E_{z}$ components if we move along a certain direction which in this case is the North direction. One important point is the independency of the values to the shower core. This is very important in real experiments. The orientation of these patterns depends on the propagating direction of an air showers as we mentioned earlier so we will have different shapes for different inclined air showers but in all circumstances we will have well distributed values in an entire radio detection area.

\section{Conclusions}

Based on simulations with CORSIKA and COREAS code we compared vertical and inclined air showers to find the best type of cosmic ray air showers to radio detection. By comparing raw radio pulses it is clear that inclined air shower can be detected in a much wider time window compared to vertical air showers which is important in real experiments. Although they may provide weaker radio pulses in comparison to the vertical ones, but the consistency of their values makes them more favorable to radio detection. We showed that for an inclined air shower with $\theta=60^{\circ}$ we can expect to measure almost the same values for total field strength 100 meter away from shower core and even further. For vertical showers we calculate nearly $60 \%$ decrease in total field component radio amplitude values just by moving $100 \mathrm{~m}$ away from shower core. This situation doesn't happen for inclined air showers where we may calculate almost the same values for total field component at the canter and at 100 meter distance from shower core.

Same scenario happened when we compared radio signal patterns in an entire radio detection area. To achieve this we used a specially designed computer code to calculate peak radio amplitude values for different electric filed components in the 32-64 MHz frequency band. It was clear from those patterns that the dependency to the shower core doesn't exist for inclined air showers which means we can expect to record even stronger radio pulses by moving along a specific direction which depends on some shower property such as the zenith angle of an air shower. The orientation of these patterns aren't the same for all kind of inclined air showers but in all situations radio antennas have detected more expanded and unified peak radio amplitude values in an entire radio detection area. We conclude from the result represented here from raw time-dependent radio pulses to filtered peak radio amplitude patterns that inclined ultra-high energy cosmic ray air showers are the most suitable to radio detection. 


\section{References}

[1] Karl-Heinz Kampert, Alan A. Watson, Extensive air showers and ultra high-energy cosmic rays: a historical review, The European Physical Journal H August 2012, Volume 37, Issue 3, pp 359-412.

[2] H. Falcke, W. D. Apel, A. F. Badea et al., Detection and imaging of atmospheric radio flashes from cosmic ray air showers, Nature 435 (2005) 313-316.

[3] T. Huege, W. D. Apel, T. Asch et al., Radio detection of cosmic ray air showers with LOPES, Nuclear Physics B (Proc. Suppl.) 165 (2007) 341-348.

[4] D. Ardouin, A. Bell'etoile, D. Charrier et al., Radio-detection signature of high-energy cosmic rays by the CODALEMA experiment, Nucl. Instr. Meth. A 555 (2005) 148-163.

[5] A. van den Berg et al., Radio detection of high-energy cosmic rays at the Pierre Auger Observatory, in: Proceedings of the 30th ICRC, Merida, Mexico, 2007, astro-ph/0708.1709.

[6] Apel, W.D., Arteaga, J.C., Badea, A.F. et al. (The KASCADE-Grande Collaboration) 2010, The KASCADE-Grande experiment, Nuclear Instruments and Methods in Physics Research A 620, $202-$ 216.

[7] Abreu, P., Aglietta, M., Ahlers, M. et al. (The Pierre Auger Collaboration) 2012, Antennas for the Detection of Radio Emission Pulses from Cosmic-Ray induced Air Showers at the Pierre Auger Observatory, JINST 7, P10011.

[8] Petrovic, J. et al., Radio emission of highly inclined cosmic ray air showers measured with LOPES, W.D., Asch, T. et al. (The LOPES Collaboration) 2007, Astronomy \& Astrophysics 462, 389-395.

[9] Huege, T., Falcke, H., Radio emission from cosmic ray air showers. Monte Carlo simulations, Astronomy and Astrophysics, v.430, p.779-798 (2005).

[10] Huege, T., Falcke, H., Radio emission from cosmic ray air showers: Simulation results and parametrization, 2005, Astropart. Physics 24, 116-136.

[11] Apel, W.D., Arteaga, J.C., Bähren, L. et al., Comparing LOPES measurements of air-shower radio emission with REAS 3.11 and CoREAS simulations, 2013, Astroparticle Physics 50-52, 76-91.

[12] Huege, T., Ulrich, R., Engel, R., Dependence of geosynchrotron radio emission on the energy and depth of maximum of cosmic ray showers, 2008, Astropart. Physics 30, 96-104.

[13] Sabouhi, M., Rastegarzadeh, G., A new method to determine air shower propagation direction based on radio signal patterns, 2015, in: Proceedings of the 34th ICRC, Hague, Netherlands, 2015.

[14] Huege, T., Ulrich, R., Engel, R., Monte Carlo simulations of geosynchrotron radio emission from CORSIKA-simulated air showers, 2007, Astropart. Physics 27, 392-405.

[15] D. Heck, J. Knapp, J.N. Capdevielle, G. Schatz, T. Thouw, CORSIKA: A Monte Carlo Code to Simulate Extensive Air Showers, FZKA Report 6019 (1998), Forschungszentrum.

[16] Karlsruhe (1998).T. Huege, M. Ludwig, C.W. James, Simulating radio emission from air showers with CoREAS, AIP Conf. Proc. 1535, 128-132 (2013), doi:10.1063/1.4807534.

[17] S. Ostapchenko., Non-linear screening effects in high energy hadronic interactions, Phys. Rev. D74 (2006) 014026.

[18] S. Ostapchenko., On the re-summation of enhanced Pomeron diagrams, Phys. Lett. B636 (2006) 40-45.

[19] J. Abraham, M. Aglietta, I.C. Aguirre, et al., Properties and performance of the prototype instrument for the Pierre Auger Observatory, Nucl. Instrum. Meth. A523 (2004) 50-95. 
[20] B. Bartoli et al. (ARGO-YBJ Collaboration), Evidence of a geomagnetic effect on extensive air showers detected with the ARGO-YBJ experiment, Phys. Rev. D 89, 052005 - Published 28 March 2014.

[21] F. G. Schröder, Instruments and Methods for the Radio Detection of High Energy Cosmic Rays, Springer Theses, DOI: 10.1007/978-3-642-33660-7_2, (C) Springer-Verlag Berlin Heidelberg 2012. 